\title{
Giving up on everything: a case and review of pervasive refusal syndrome
}

\author{
Jordan Ho, Brynn Charron
}

\begin{abstract}
We present a case of pervasive refusal syndrome (PRS), in which a 11-year-old female patient refused to eat, drink, interact, or self-care in any way. The patient was kept on IV nutrition and multiple therapeutic options were tried to no effect. After 18 months, the patient spontaneously recovered with no lasting harm, and her symptoms did not recur. We further discuss the current state of clinical knowledge on PRS, as well as the form that has appeared amongst children in asylum-seeking families in Sweden.
\end{abstract}

\section{CASE PRESENTATION}

The following is a summary of a case by McNicholas et al. about a patient in Ireland. ${ }^{1}$

An 11-year-old girl was referred to a paediatric hospital with concerns over an 8-week decline in well-being of unknown cause, in which the patient refused to eat or drink and seemed disengaged. This episode began with a 2-week history of non-productive cough that did not improve with over-the-counter medication, prompting her parents to consult their family practitioner. A chest x-ray demonstrated hyperinflated lungs which did not improve after a 3-week course of oral steroids. The patient then began to experience colicky abdominal pain and a disturbed sleep schedule, and spent most of her days "moaning and lying about." At this point, the patient was unable to attend school and had lost significant weight.

The patient's past medical history was not significant, and the physical examination was unremarkable. All investigations proved inconclusive, including a complete blood count, urea, electrolytes, amylase, liver function tests, bone profile, thyroid function tests, venous blood gases, abdominal ultrasound, and computed tomography (CT) scan of the brain. Two electroencephalograms (EEGs) (under usual and sleep-deprived protocols) demonstrated generalised discharges which were asymptomatic.

The patient was the youngest of 3 children. There was no history of abuse or marital discord in the family. Her parents were authoritative, and her siblings were high-functioning and welladjusted. There was a distant family history of depression. The patient's developmental history was normal; however, she was described as "a bit anxious" by her parents, especially with regards to her health. The patient was also described by her parents as "a sensitive child" and had no close friends, but appeared socially engaged. The patient had no difficulties in school, and there was no history of bullying.

On admission, the patient was communicative to a limited degree but was preoccupied with her physical symptoms. As time progressed, the patient became increasingly disengaged from family and staff. On rare occasions, she communicated concerns that her parents were infantilizing her. Over the next few months, all communication and food intake from the patient stopped, requiring a nasogastric feeding tube to be established.

Duringbiweekly sessions with a clinical psychologist, the patient remained silent and rigid but appeared aware of the conversation. The utility of these sessions eventually became restricted due to the patient covering her face, preventing any insight into her thoughts. During family sessions, the patient refused to participate and often tried to appear disinterested by staring intensely. Other attempted interventions included art psychotherapy, physiotherapy, hydrotherapy, and antidepressants and antipsychotics (sertraline $150 \mathrm{mg}$, paroxetine $20 \mathrm{mg}$, venlafaxine $150 \mathrm{mg}$, risperidone $2 \mathrm{mg}$ ), all with no discernible effect. Lorazepam $4 \mathrm{mg}$ was given for extreme agitation only. Electroconvulsive therapy (ECT) was rejected by the parents.

After 18 months in hospital, the patient had shown no response to any intervention and refused to eat, drink, engage in self-care, or communicate. As a result, the decision was made to transfer the patient to a child psychiatry inpatient unit abroad.

\section{CASE OUTCOME}

Two days before the transfer, the patient wrote a note requesting to staff that she "wanted to go home so ring my mammy." The following day, she went clothes-shopping with her family, ate at a fast food restaurant, and began to communicate through notewriting. Within 2 weeks she was speaking fluently, and 4 weeks after discharge, she was fully integrated back at school.

The patient refused to attend follow-up appointments with the psychiatry team. She continued to do well at school, made new friends, and has been described as "having good coping skills" and "very happy". Six years on, she has remained physically well and has gone on to university.

\section{DISCUSSION}

\section{Pervasive Refusal Syndrome}

Pervasive refusal syndrome (PRS) was first described in 1991 by Bryan Lask and his colleagues as a potentially lifethreatening condition involving profound and pervasive refusal to sustain basic functions of life, such as eating, drinking, or communicating. ${ }^{2}$ Since then, less than 30 cases of PRS have been described in the literature, both due to the rarity of the syndrome and uncertainty over its diagnostic criteria. The etiology of PRS is currently still unknown, and as the behaviour does not seem to 
occur with any conscious intention, theories for its causes include conscientious "perfectionist" personality, an extreme presentation of learned helplessness, and conditioned response to trauma and environmental stressors. ${ }^{3,4}$ PRS has not been included in the Diagnostic and Statistical Manual of Mental Disorders 5 (DSM-5) or the International Statistical Classification of Disease and Related Health Problems 10th Revision (ICD-10), and the current diagnostic criteria for PRS is quite broad: ${ }^{4}$

- Partial or complete refusal in three or more of: eating, mobilisation, speech, attention to personal care

- Active and angry resistance to acts of help and encouragement

- Social withdrawal and school refusal

- No organic condition accounts for the severity of the degree of symptoms

- No other psychiatric disorder that could better account for the symptoms

- The endangered state of the patient requires hospitalisation

The generally accepted treatment for this condition, laid out by Nunn et al. in 1998, requires a multidisciplinary team approach, including nurses, paediatricians, dieticians, physiotherapists, and psychiatrists employing both individual and family-based psychotherapy techniques. ${ }^{5,6}$ Management and recovery from PRS is regarded as a long-term process. This case follows the generally accepted treatment regimen, although in this case, the girl recovered much more quickly - the original cases described a "slow consistent progress" towards recovery.

The differential diagnosis for this case included major depressive disorder, anorexia nervosa, selective mutism, school refusal, conversion disorder, somatisation disorder, and chronic fatigue syndrome - although all were considered to be inadequate descriptions of the presentation. ${ }^{1}$ Catatonia was also on the differential for this case, which would have had significant implications on treatment and thus warranted careful consideration. Catatonia is described in the ICD-10 as "a disorder of diminished (stupor) ... psychomotor activity associated with catatonic symptoms," and the DSM-5 lists 5 clinical features: motor immobility, excessive motor activity, extreme negativism, peculiarities of voluntary movement, and echolalia or echopraxia., ${ }^{8,9}$ As some of these features are contradictory (eg. motor immobility and excessive motor activity), it has been suggested that only 2 motor signs in a 24-hour period are required to make the diagnosis. ${ }^{10}$ Current treatment for catatonia includes benzodiazepines, high dose barbiturates, and ECT. ${ }^{11}$ In this case, the parents did not support the usage of ECT to treat their child, and the medications did not appear to help the patient.

PRS has also been reconceptualised as "pervasive arousalwithdrawal syndrome," based on a neurobiological explanatory model based on hyperarousal of the autonomic nervous system. In this model, it is proposed that both the sympathetic and parasympathetic nervous systems are hyperactivated, resulting in exhaustion and refusal to care for oneself. The model would support treating PRS using strategies that inhibit gamma-aminobutyric acid (GABA), such as valproate, to calm both systems at once. ${ }^{12}$

\section{Resignation Syndrome}

The literature also describes many cases of patients with PRSlike symptoms, although the cases were not formally diagnosed as PRS. The most substantive collection of these cases comes from Sweden, where in 2004, Bodegård described 23 such cases in children from traumatized asylum-seeking families. ${ }^{13}$ These children were "totally passive, immobile, lacking tonus, withdrawn, mute, unable to eat or drink, incontinent, and [unreactive] to physical stimuli or pain," and he considered the most serious cases of this to be "identical to PRS." Secondary symptoms included "tachycardia, increased temperature, weight gain, edema, profuse sweating, reactivation of latent viral infection, skin ulcers, and muscular atrophy."13

Of the 23 cases, half were of Asian ethic background, most were the first-born, half had attempted suicide, all had developed symptoms after arriving in Sweden, all were considered political refugees, and none of their siblings had developed similar symptoms. Furthermore, half of the families had been given deportation orders before the symptoms started, and the other half were still undergoing review by the Migration Board. ${ }^{13}$

With respect to treatment, all were given the treatment recommended for PRS. It was noted that "achievement of a permanent residence permit was necessary for restoration of basic life security ... [and] seemed to be a condition for successful treatment." although treatment had to continue for many months before full restoration. Of the 23 children, 17 achieved residence permits on the basis of humanitarian grounds for their children, and all recovered successfully. ${ }^{13}$

This condition, although not formally designated PRS in the literature, was further described in more than 400 adolescents ages 7-19 in Sweden between 2002 and 2006, and was given several different names, including "uppgivenhetssyndrom" or "resignation syndrome," "depressive devitalisation," and "apathetic syndrome," to specifically describe the occurrence of PRS-like symptoms in adolescents in refugee-seeking families. ${ }^{14}$ Further investigations have included neuroradiology, toxicology screening, anamnestic interviewing, neurophysiological examination, lumbar puncture, EEG, and CT of the brain, all of which have been unremarkable..$^{13,15,16}$ All described cases belonged to a political or ethnic minority, of which those of Uighur background were particularly affected. Traumatisation in terms of physical abuse or witnessing of abuse in the close family were described in half of the individuals. ${ }^{17}$

Cases of resignation syndrome have continued to be described in the literature as far as 2014; however, no cases have been described outside of Sweden. The prevalence of the syndrome in Sweden was calculated to be about $2 \%$ of the children from all asylum-seeking families. ${ }^{16,17}$

\section{CONCLUSION}

PRS and its various namesakes are poorly understood syndromes that occur in adolescents, all characterized by a refusal to eat, sleep, interact or self-care in any way. A form of PRS called resignation syndrome has been reported extensively in Sweden, in children from asylum-seeking families who had either pending or rejected residence permits, with a prevalence of around $2 \%$. 
The presence of these cases in Sweden speaks to the trauma that asylum-seeking families face both before and during their migration process. More work is required to understand the etiology and pathophysiology behind pervasive refusal syndrome.

\section{REFERENCES}

1. McNicholas F, Prior C, Bates G. A case of pervasive refusal syndrome: a diagnostic conundrum. Clin Child Psychol P. 2012 Jul;18(1):137-50. https://doi.org/10.1177/1359104512440079

2. Lask B, Britten C, Kroll L, et al. Children with pervasive refusal. Arch Dis Childhood. 1991 Jul;66:866-9. https://doi.org/10.1136/adc.66.7.866

3. Nunn KP, Thompson SL. The pervasive refusal syndrome: learned helplessness and hopelessness. Clin Child Psychol P. 1996 Jan;1(1):12132. https://doi.org/10.1177/1359104596011011

4. Jaspers T, Hanssen GMJ, van der Valk JA, et al. Pervasive refusal syndrome as part of the refusal-withdrawal-regression spectrum: critical review of the literature illustrated by a case report. Eur Child Adolesc Psychiatry. 2009 Nov;18(11):645-51. https://doi.org/10.1007/ s00787-009-0027-6

5. Nunn KP, Thompson SL, Moore SG, et al. Managing pervasive refusal syndrome: strategies of hope. Clin Child Psychol P. 1998 Apr;3(2):22949. https://doi.org/10.1177/1359104598032007

6. von Schoen-Angerer T, Helmschmidt E, Madeleyn R, et al. A general pediatrics and integrative medicine approach to pervasive refusal syndrome: a case report. Perm J. 2016 Fall;20(4):112-5. https://doi. org/10.7812/TPP/15-238

7. Thompson SL, Nunn KP. The pervasive refusal syndrome: the RAHC experience. Clin Child Psychol P. 1997 Jan;2(1):145-65. https://doi. org $/ 10.1177 / 1359104597021010$

8. Diagnostic and statistical manual of mental disorders. 5th ed. Arlington: American Psychiatric Association; 2013. Chapter 9, Somatic symptom and related disorders. https://doi.org/10.1176/appi. books. $9780890425596 . \mathrm{dsm} 09$

9. Organic catatonic disorder [Internet]. Geneva: World Health Organization; 2016 [cited 2018 Dec 19]. Available from: https://icd.who. int/browse10/2016/en\#/F06.1

10. Penland HR, Weder N, Tampi RR. The catatonic dilemma expanded. Ann Gen Psych. 2006 Sep;7(5):14-22. https://doi.org/10.1186/1744859X-5-14

11. Taylor MA, Fink M. Catatonia in psychiatric classification: a home of its own. Am J Psych. 2003 Jul;160(7):1233-41. https://doi.org/10.1176/ appi.ajp.160.7.1233

12. Nunn KP, Lask B, Owen I. Pervasive refusal syndrome (PRS) 21 years on: a re-conceptualisation and a renaming. Eur Child Adolesc Psychiatry. 2014 Mar;23:163-72. https://doi.org/10.1007/s00787-0130433-7

13. Bodegård G. Pervasive loss of function in asylum-seeking children in Sweden. Acta Paediatrica. 2004;94(12):1706-7. https://doi. org/10.1111/j.1651-2227.2005.tb01841.x

14. Thomas SP. Resignation syndrome: is it a new phenomenon or is it catatonia? Iss Men Heal Nurs. 2017 Jul;38(7):531-2. https://doi.org/10.1 $\underline{080 / 01612840.2017 .1341229}$

15. Sallin K, Lagercrantz H, Evers K, et al. Resignation syndrome: Catatonia? Culture-bound? Front Behav Neurosci. 2016 Jan;10:7. https://doi.org/10.3389/fnbeh.2016.00007

16. Forslund C, Johansson BA. Pervasive refusal syndrome among inpatient asylum-seeking children and adolescents: a follow-up study. Eur Child Adolesc Psych. 2013 Apr;22:251-8. https://doi.org/10.1007/ $\underline{\text { s00787-012-0341-2 }}$
17. Bodegård G. Comment on the paper "Pervasive refusal syndrome (PRS) 21 years on - a reconceptualization and renaming" by Ken Nunn, Bryan Lask, and Isabel Owen. Eur Child Adolesc Psychiatry. 2014 Mar;23:179-81. https://doi.org/10.1007/s00787-013-0435-5 